\title{
COMPLETE AMONOTONIC DECOMPOSITIONS OF COMPACT CONTINUA
}

\author{
HARVY LEE BAKER, JR. ${ }^{1}$
}

A collection $G$ of sets will be called amonotonic if and only if no set of $G$ is a subset of another one. A complete amonotonic subcollection of a collection $G$ of sets is a maximal amonotonic subcollection of $G$. It follows from the axiom of choice that every collection of sets contains a complete amonotonic subcollection. Similar definitions can be given of course for any partially ordered system. The collection of all subcontinua of a continuum (a closed and connected set) $M$ will be denoted by $C(M)$, and a complete amonotonic subcollection of $C(M)$ which contains more than one element will be called a complete amonotonic decomposition of $M$. A study is begun of complete amonotonic decompositions of compact metric continua. It is shown that no compact metric continuum has a finite complete amonotonic decomposition and that every such decomposition of an arc is uncountable. Countable complete amonotonic decompositions of compact metric continua however are possible (e.g. the set of all arcs of a circle which are irreducible about a given Cantor set lying on that circle), and the primary result obtained here is a characterization of those compact metric continua $X$ for which there is a complete amonotonic decomposition $G$ such that

(1) $g+g^{\prime}=X$ for arbitrary elements $g$ and $g^{\prime}$ in $G$, and

(2) $X-g$ is connected for every $g$ in $G$. Both properties are possessed by the above amonotonic decomposition of a circle, and (1) implies that $G$ is countable. The characterization given involves the notion of cyclotomic subsets of a continuum as introduced by R. L. Moore. A definition will be given here in $\$ 2$ of an atomic decomposition of a space and certain restrictions placed upon sets appearing in the definition yield the definitions of Moore.

1. The following two theorems are fundamental in the study of complete amonotonic decompositions of continua. It is interesting to note that the second one implies that it is false that if $C(X)$ is homeomorphic to $C\left(X^{\prime}\right)$ in the Hausdorff metric topology then there is a countable complete amonotonic decomposition of $X$ if and only if

Presented to the Society, January 27, 1965 under the title Complete amonotonic collections of subcontinua of a compact continuum; received by the editors March 24, 1966.

1 This paper is drawn from the author's doctoral dissertation, directed by Professor R. L. Moore at the University of Texas, 1965. 
there is such a decomposition of $X^{\prime}$. For if we let $X$ and $X^{\prime}$ be an arc and a simple closed curve respectively, then $C(X)$ is homeomorphic to $C\left(X^{\prime}\right)$, but $X^{\prime}$ has a countable complete amonotonic decomposition whereas $X$ does not.

In the theorems and lemmas of this section as well as of the next one, $X$ denotes a compact metric continuum.

TheOREM 1.1. There does not exist a finite complete amonotonic decomposition of $X$.

Proof. Suppose that $G$ is a finite complete amonotonic decomposition of $X$ and that $M_{1}$ and $M_{2}$ are two elements of $G$ which have a common point $Q$. Let $D$ denote an open set relative to $M_{1}$ such that (1) $D$ intersects every element of $G$ which intersects $M_{1}-M_{1} \cdot M_{2}$, and (2) $\bar{D} \cdot M_{2}=0$. Let $T_{1}$ denote the component of $M_{1}-D$ which contains $Q . T_{1}$ intersects both $M_{1} \cdot M_{2}$ and $\bar{D}$ but contains no point of $D$. Let $T_{2}$ denote a subcontinuum of $M_{2}$ which contains $Q$ and intersects $M_{2}-M_{2} \cdot M_{1}$ but does not contain all of it. Since $T_{1}+T_{2}$ contains no element of $G$, some element of $G$ contains it. If $N_{1}$ is one such element then there exists a subcontinuum $T_{3}$ of $M_{2}$ which contains $Q+T_{2}$, intersects $M_{2}-M_{2} \cdot N_{1}$, and does not contain all of $M_{2}-N_{1} \cdot M_{2}$.

Continuing in this manner, the process will terminate in a finite number of steps, say $e-1$. The subcontinuum $T_{1}+T_{2}+\cdots+T_{e}$ of $X$ neither contains nor is a subset of any element of $G$. The argument for this is routine.

THEOREM 1.2. Every complete amonotonic decomposition of an arc is uncountable.

Proof. The triangular disc with vertices $(0,0),(0,1),(1,1)$ may be considered to be $C([0,1])$, and a proper translation of the above theorem in to this space yields a question which is easily answered. Another proof stems from the fact that if $G$ is a complete amonotonic decomposition of $[0,1]$ and $I_{1}, I_{2}$ are in $G$ then there is an element $I_{3}$ of $G$ such that $G L B\left(I_{3}\right)$ is between $G L B\left(I_{1}\right)$ and $G L B\left(I_{2}\right)$.

2. In [4] R. L. Moore gives definitions of a graphatomic subset, of an arcatomic subset, and of a dendratomic subset of a continuum. The following definition is based upon those given by Moore and in the case of the three terms mentioned above is equivalent when various restrictions are placed on the collection $W$ mentioned in it.

Definition 2.1. Suppose $S$ is a topological space, $G$ is an upper semicontinuous decomposition of $S$ into mutually exclusive continua, 
and $W$ is a collection of topological spaces (not necessarily related to $S$ ) such that:

(a) $S / G$ is homeomorphic to an element of $W$,

(b) if $H$ is an u.s.c. decomposition of $S$ into mutually exclusive continua and $S / H$ is homeomorphic to an element of $W$ then $G$ refines $H$.

Then $G$ is said to be a $W$-atomic decomposition of $S$.

If $W$ consists of a single arc then the elements of $G$ are called (by Moore) arcatomic subsets of $S$. Similar definitions are made by Moore for cyclatomic subsets, dendratomic subsets, and graphatomic subsets ("graph" being substituted here for "compact metric locally connected continuum"). The definition of a cyclatomic subset does not occur in [4] but Moore has used the term in his classes, and its definition is clear if one knows the definitions of the other atomic subsets. Examples of theorems established by Moore are the following:

Theorem $\mathrm{M}_{1}$. A compact metric continuum has a dendratomic subset if and only if it is not a web.

TheOREM $\mathrm{M}_{2}$. If the compact metric continuum $M$ is not a triod it has a dendratomic subset and if it has more than one of them they are its arcatomic subsets.

TheOREM $\mathrm{M}_{3}$. Every compact metric continuum which is irreducible between two points has a dendratomic subset and if it has more than one of them they are its arcatomic subsets.

Definition 2.2. A proper domain is an open set whose complement is connected.

Definition 2.3. A cyclatomic subset of type I of a continuum $M$ is a cyclatomic subset $x$ of $M$ such that if $t$ is another cyclatomic subset of $M$ and $T$ is a component of $M-(x+t)$ then $\bar{T}$ is irreducible from $x$ to $t$.

The following four lemmas are presented without proof. Recall that $X$ denotes a compact metric continuum. The first two may be proved in a straightforward manner. The third seems as though it should follow from existing literature, but the author was driven to a lengthy analysis (which presented no special difficulties) in order to prove it. The fourth follows directly from Theorem 53, Chapter V of [4] (Theorem $\mathrm{M}_{3}$ ).

Lemma 2.1. If $D$ and $D^{\prime}$ are mutually exclusive proper domains and $T$ is a component of $X-\left(D+D^{\prime}\right)$ then $T$ intersects both $\bar{D}$ and $\bar{D}^{\prime}$.

Lemma 2.2. If $D, D^{\prime}$ and $D^{\prime \prime}$ are proper domains and $X-\left(D+D^{\prime}\right)$ $=H+K$ where $H$ and $K$ are mutually separated and $H$ contains $D^{\prime \prime}$, and $T$ is a component of $H-D^{\prime \prime}$ then $T$ intersects $\bar{D}+\bar{D}^{\prime}$. 
Lemma 2.3. $X$ is a simple closed curve if and only if there is a countable dense subset of $X$, say $T$, such that $X-T$ is totally disconnected and such that if $P$ and $Q$ are distinct points of $T$ then $X-(P+Q)$ is the sum of two mutually separated connected sets.

Lemma 2.4. If $X$ is irreducible about two points and there is an upper semicontinuous decomposition $G$ of $X$ into mutually exclusive continua such that $X / G$ is an arc, then $X$ has arcatomic subsets.

In the following theorem $G$ denotes a collection of mutually exclusive connected proper domains of $X$, and $H$ denotes the collection of complements of the elements of $G$.

TheOREM 2.1. Suppose that $H$ is a complete amonotonic decomposition of $x$ and that $W$ is a collection to which $x$ belongs if and only if either (1) $x$ is a component of $X-G^{*}$ (the sum of the elements of $G$ ) and $x \cdot \bar{g}=0$ for every $g$ in $G$, or (2) for some $g$ in $G x=g+$ all the components of $X-G^{*}$ which intersect $\bar{g}$. Then $W$ is u.s.c. and $X / W$ is a simple closed curve.

Proof. Let $W^{\prime}$ and $W^{\prime \prime}$ be the subcollections of $W$ described by (1) and (2) respectively. Suppose $g_{1}$ and $g_{2}$ are distinct elements of $G$. The following things will be shown:

(a) $X-\left(g_{1}+g_{2}\right)$ is not connected,

(b) $\bar{g}_{1} \cdot \bar{g}_{2}=0$,

(c) $X-\left(g_{1}+g_{2}\right)$ is the sum of two continua,

(d) $w \cdot w^{\prime}=0$ for distinct elements $w$ and $w^{\prime}$ of $W$,

(e) $W$ is u.s.c.,

(f) $X-\left(h_{1}+h_{2}\right)$ is the sum of two mutually separated connected sets, where $h_{i}$ is the element of $W^{\prime \prime}$ containing $g_{i}$, and

(g) $X / G$ is a simple closed curve.

It follows that (a) is true since if $D$ and $D^{\prime}$ are open sets whose closures lie in $g_{1}$ and $g_{2}$ respectively, and there is a component $C$ of $X-\left(D+D^{\prime}\right)$ which contains $X-\left(g_{1}+g_{2}\right)$, then $C$ neither contains nor is a subset of any element of $H$.

Suppose now that $\bar{g}_{1} \cdot \bar{g}_{2} \neq 0$. There is a component $T$ of $X-\left(\bar{g}_{1}+\bar{g}_{2}\right)$, since $G$ cannot be finite. If $T$ is the only component of $X-\left(g_{1}+g_{2}\right)$ which intersects $X-\left(\bar{g}_{1}+\bar{g}_{2}\right)$ then by a construction similar to the one above for (a), it follows that $H$ is not a complete amonotonic decomposition of $X$. Hence there are two components $T_{1}$ and $T_{2}$ of $X-\left(g_{1}+g_{2}\right)$ which intersect $X-\left(\bar{g}_{1}+\bar{g}_{2}\right)$. Let $K_{1}$ and $K_{2}$ be two mutually exclusive closed point sets whose sum is $X-\left(g_{1}+g_{2}\right)$ and which contain $T_{1}$ and $T_{2}$ respectively. Suppose that $K_{n} \cdot G^{*}=0$ for $n=1$ or 2 . Then the complement of the set $K_{n}-K_{n}\left(\bar{g}_{1}+\bar{g}_{2}\right)$ is a subcontinuum 
of $X$ whose complement does not intersect $G^{*}$ and thus it neither contains nor is a subset of any element of $H$. This is a contradiction. Let $t_{1}$ and $t_{2}$ be elements of $G$ which lie in $K_{1}$ and $K_{2}$ respectively. From Lemma 2.2 it follows that if $x$ is a point of $K_{1}-t_{1}$ the component of $K_{1}-t_{1}$ containing $x$ intersects $\bar{g}_{1}+\bar{g}_{2}$. Furthermore since $\bar{g}_{1} \cdot \bar{g}_{2} \neq 0$ and no point of this set belongs to $t_{1}+t_{2}$, it follows that $X-\left(t_{1}+t_{2}\right)$ is a continuum. This contradicts (a). Hence (b) follows.

Suppose now that $X-\left(g_{1}+g_{2}\right)$ has at least three components. Let $H_{1}, H_{2}$ and $H_{3}$ be three mutually exclusive closed point sets whose sum is $X-\left(g_{1}+g_{2}\right)$. If $n \leqq 3$ and $H_{n}$ does not intersect $G^{*}$ then, as was shown previously in a similar case, $X-\left(H_{n}-H_{n} \cdot\left(\bar{g}_{1}+\bar{g}_{2}\right)\right)$ is a continuum which neither contains nor is a subset of an element of $H$. But this involves a contradiction, and hence each of $H_{1}, H_{2}$ and $H_{3}$ intersects $G^{*}$. Let $U$ and $U^{\prime}$ be elements of $G$ which lie in $H_{1}$ and $H_{2}$ respectively. With the aid of Lemmas 2.1 and 2.2 it follows that $M-\left(t_{1}+t_{2}\right)$ is connected. Again, this contradicts (a) and hence (c) follows.

Suppose a component $T$ of $M-G^{*}$ intersects the closure of two elements $g_{1}$ and $g_{2}$ of $G$. Let $U$ and $V$ denote two mutually exclusive continua whose sum is $X-\left(g_{1}+g_{2}\right)$ and suppose $U$ contains $T$. Let $t_{1}$ and $t_{2}$ denote elements of $G$ lying in $U$ and $V$ respectively. Now $t_{1}$ does not intersect $T$ and by Lemma 2.3 it follows that $M-\left(t_{1}+t_{2}\right)$ is connected. Hence (d) follows.

(e) is reasonably clear and (f) follows from an argument very similar to that given for (c). (g) now follows from Lemma 2.3.

THEOREM 2.2. Under the same hypothesis (and with the same terminology) as the previous theorem, $X$ has cyclatomic subsets of type $I$.

Proof. Suppose $w$ and $w^{\prime}$ are distinct elements of $W^{\prime \prime}$, and that $T_{1}$ and $T_{2}$ are the two mutually separated sets such that $T_{1}+T_{2}=X$ - $\left(w+w^{\prime}\right)$. If $\bar{T}_{1} \cdot \bar{T}_{2} \neq 0$ then $\bar{T}_{1}+\bar{T}_{2}$ is connected, and since, as is easy to show, $\bar{T}_{1}+\bar{T}_{2}=X-\left(D_{1}+D_{2}\right)$ where $D_{1}, D_{2} \in G$ and lie in $w$ and $w^{\prime}$ respectively, it follows that (a) of Theorem 2.1 is contradicted. $\bar{D}_{1}$ is irreducible from $\bar{D}_{1} \cdot \bar{T}_{1}$ to $\bar{D}_{1} \cdot \bar{T}_{2}$, for otherwise a continuum could be constructed whose complement lies in $D_{1}+D_{2}$ but which contains neither $D_{1}$ nor $D_{2}$. (If $N \subset \bar{D}_{1}$ and is connected, and $D$ is an open set such that $\bar{D} \subset D_{2}$ then the component of $X-\left(D+\left(D_{1}-D_{1} \cdot N\right)\right)$ which contains $N+\bar{T}_{1}+\bar{T}_{2}$ is such a continuum if $N \neq \bar{D}_{1}$.)

Using the two results above together with the fact that if $x \in W^{\prime}$ then $\bar{X}-\bar{x}=X$ it is easily established that if $x_{1}$ and $x_{2}$ are distinct elements of $W^{\prime}$ and $T_{1}$ is a component of $X-\left(x_{1}+x_{2}\right)$ then $\bar{T}_{1}$ is irreducible from $\bar{T}_{1} \cdot x_{1}$ to $\bar{T}_{1} \cdot x_{2}$. Now the set $\left\{\bar{T}_{1} \cdot x_{1}, \bar{T}_{1} \cdot x_{2}\right\}$ 
$+\left\{y: y \subset \bar{T}_{1}\right.$ and $\left.y \in W\right\}$ is an u.s.c. decomposition of $\bar{T}_{1}$ into an arc, and hence from Lemma 2.4 $\bar{T}_{1}$ has arcatomic subsets. Let $A_{1}$ and $A_{2}$ denote the collections of arcatomic subset of $\bar{T}_{1}$ and $\bar{T}_{2}$ respectively. The set $\left\{x: x \in A_{1}+A_{2}\right.$, and $\left.0=x \cdot w+w^{\prime} \cdot x\right\}+\left\{w, w^{\prime}\right\}$ is a cyclatomic collection which fills up $X$. A detailed proof that this collection is a cyclatomic one will not be given.

THEOREM 2.3. A necessary and sufficient condition that $X$ have cyclatomic subsets of type $I$ is that there exists a complete amonotonic decomposition $H$ of $X$ such that (1) $h_{1}+h_{2}=X$ for distinct elements $h_{1}, h_{2}$ of $H$, and (2) $X-h$ is connected for every $h$ in $H$.

Proof. Suppose $X$ has cyclatomic subsets of type I. Let $W$ be a complete amonotonic decomposition of $X / G$ which has properties (1) and (2) described in the paragraph preceding section one. Let $W^{\prime}$ be the collection of all sets $T^{-1}(W)$ where $w \in W$ and $T$ is the natural map from $X$ into $X / G$. $W^{\prime}$ is a complete amonotonic decomposition of $X$ which has properties (1) and (2). The converse of what has just been shown is Theorem 2.2 and hence the above theorem follows.

Theorem 2.3 may be stated in the following slightly different form.

THEOREM 2.4. There exists a complete amonotonic decomposition $H$ of $X$ satisfying (1) and (2) of Theorem 2.3 if and only if there is a monotone continuous map from $X$ onto a simple closed curve such that if $X^{\prime}$ is a proper closed subset of $X$ then $f\left(X^{\prime}\right) \neq C$.

3. Numerous studies have been made of the lattice of closed subsets of a topological space $X$, as well as of $C(X)$. Dilworth in [3], for example, defines an independent subset of a partially ordered set and speaks of a maximal independent set (finite in his paper). These definitions are equivalent to the set-theoretic definitions given here, although the work of Dilworth is not related to the work presented here. A similar statement seems also to apply to most of the work concerning the lattice of closed subsets of $X$. There seems to be two areas of interest concerning complete amonotonic decompositions, one of which does have some bearing on the study of the lattice of closed subsets of $X$.

First, there arises the problem of characterizing those continua which have countable complete amonotonic decompositions. The author has shown (reported in [1] and [2]) that connected finite graphs with no end points have property $C$ (that is, have countable complete amonotonic decompositions), that a wide variety of continua which includes all compact $n$-manifolds with or without boundary $(n>1)$ and many other continua have property $C$. 
Secondly, the problem of characterizing continua in terms of their amonotonic decompositions seems to be of interest. In [2] the author reported the following result:

Theorem. The compact metric continuum $X$ is a simple closed curve if and only if there is a countable complete amonotonic decomposition of $X$, each element of which is an arc.

Arcs and dendrons can also be characterized by their complete amonotonic decompositions into arcs (e.g., if each two arcs of the decomposition intersect and $X$ is not a simple closed curve, then $X$ is an arc). Higher dimensional analogs of such characterizations would seem to be of interest.

\section{REFERENCES}

1. H. L. Baker, Jr., Complete amonotonic collections of subcontinua of a compact continuum, Abstract 619-119, Notices Amer. Math. Soc. 12 (1965), 91.

2. - Concerning complete amonotonic collections of subcontinua of a compact continuum, Abstract 626-24, Notices Amer. Math. Soc. 12 (1965), 697.

3. R. P. Dilworth, A decomposition theorem for partially ordered sets, Ann. of Math. 51 (1950), 161-166.

4. R. L. Moore, Foundations of point set theory, rev. ed., Amer. Math. Soc. Colloq. Publ. Vol. 13, Amer. Math. Soc., Providence, R. I., 1963.

UNIVERSity OF Nebraska 